\title{
Correction to: Hyper-response to Novelty Increases c-Fos Expression in the Hippocampus and Prefrontal Cortex in a Rat Model of Schizophrenia
}

\author{
Tomas Monfil ${ }^{1,2}$ - Rubén Antonio Vázquez Roque ${ }^{3}$ Israel Camacho-Abrego ${ }^{3} \cdot$ Hiram Tendilla-Beltran $^{3}$. \\ Tommaso lannitti ${ }^{4} \cdot$ Ivan Meneses-Morales ${ }^{5} \cdot$ Patricia Aguilar-Alonso $^{6} \cdot$ Gonzalo Flores $^{3}{ }^{3}$. \\ Julio Cesar Morales-Medina ${ }^{1}$ (1)
}

Published online: 13 January 2018

(c) Springer Science+Business Media, LLC, part of Springer Nature 2018

\section{Correction to: Neurochem Res \\ https://doi.org/10.1007/s11064-017-2439-x}

The original version of this article unfortunately contained a mistake. The spelling of the author Tommaso Ianniti was incorrect and has been corrected as Tommaso Iannitti. The original article has been corrected.

The original article can be found online at https://doi.org/10.1007/ s11064-017-2439-x.

\section{Gonzalo Flores} gonzaloflores56@gmail.com; gonzalo.flores@correo.buap.mx

$\triangle$ Julio Cesar Morales-Medina jmoralesm@cinvestav.mx

1 Centro de Investigación en Reproducción Animal, CINVESTAV-UAT, AP 62, 90000 Tlaxcala, Mexico

2 Departamento de Fisiología, Biofísica y Neurociencias, CINVESTAV, 07360 Ciudad de Mexico, Mexico

3 Lab. Neuropsiquiatría, Instituto de Fisiología, Benemérita Universidad Autónoma de Puebla, 14 Sur 6301, CP 72570 Puebla, Mexico

4 KWS BioTest, Marine View Office Park, Portishead, Somerset BS20 7AW, UK

5 Departamento de Bioquímica, Facultad de Medicina, Universidad Nacional Autónoma de México, 04510 Ciudad de Mexico, Mexico

6 Facultad de Ciencias Químicas, Benemérita Universidad Autónoma de Puebla, 72570 Puebla, Mexico 